\title{
Fabrication of Electrospun Eugenol/Cyclodextrin Inclusion Complex Nanofibrous Webs for Enhanced Antioxidant Property, Water Solubility, and High Temperature Stability
}

\author{
Asli Celebioglu, ${ }^{\dagger}$ Zehra Irem Yildiz, ${ }^{\dagger}$ and Tamer Uyar* ${ }^{\dagger} \uparrow$ \\ ${ }^{\dagger}$ Institute of Materials Science \& Nanotechnology, UNAM-National Nanotechnology Research Center, Bilkent University, Ankara \\ 06800, Turkey
}

Supporting Information

\begin{abstract}
In this study, inclusion complexes (IC) of three cyclodextrin derivatives (HP- $\beta$-CD, HP- $\gamma$-CD, and M- $\beta$-CD) with eugenol (essential oil compound) were formed in highly concentrated aqueous solutions and then transformed into selfstanding functional nanofibrous webs by electrospinning. The improved aqueous solubility of eugenol was confirmed by phase solubility diagrams, in addition, the phase solubility tests also revealed 1:1 molar ratio complexation between host:guest molecules; CD:eugenol. Even though eugenol has a volatile nature, a large amount of eugenol ( 70-95\%) was preserved in eugenol/cyclodextrin inclusion complex nanofibrous webs (eugenol/CD/IC-NW). Moreover, enhanced thermal stability of eugenol was recorded for eugenol/CD/IC-NW (up to $\sim 310^{\circ} \mathrm{C}$ ) when compared to pure form of eugenol (up to $\sim 200{ }^{\circ} \mathrm{C}$ ). The eugenol/CD/IC-NW exhibited fast dissolving behavior in water, contrary to poorly water-soluble eugenol. It was observed that the complexation between $\mathrm{M}-\beta$-CD and eugenol was the strongest when compared to other two host CD molecules (HP$\beta$-CD and HP- $\gamma$-CD) for eugenol/CD/IC-NW samples. The electrospun eugenol/CD/IC-NW samples have shown enhanced antioxidant activity compared to pure form of eugenol. In summary, cyclodextrin inclusion complexes of essential oil compounds, such as eugenol, in the form of self-standing nanofibrous webs may have potentials for food and oral-care applications due to their particularly large surface area along with fast-dissolving character, improved water solubility, high temperature stability, and enhanced antioxidant activity.
\end{abstract}

KEYWORDS: cyclodextrin, inclusion complex, electrospinning, nanofibers, eugenol, antioxidant property, essential oils

\section{INTRODUCTION}

Essential oils which are extracted from plants are composed of highly concentrated hydrophobic volatile aroma compounds, such as terpenes, terpenoids, phenol-derived aromatic components, and aliphatic components. ${ }^{1}$ Due to their specific properties, such as antioxidant, antibacterial, antifungal, antiseptic, and fragrance, etc., essential oils are often used in cosmetic, food, and medical application areas. ${ }^{1,2}$ The phenolic compounds present in essential oils are quite useful as flavoring agents for foods without known mutagenic or toxic side effects. ${ }^{3}$ Additionally, due to their pronounced antioxidant properties, these essential oil compounds have drawn attention as natural preservatives in active food packaging applications for enhanced shelf life of foods. ${ }^{1}$ Due to the safety issues in active food-packaging, the use of natural extracts of essential oils which are also classified as generally recognized as safe (GRAS) by the U.S. Food and Drug Administration (FDA), are more desirable compared to synthetic and carcinogenic antioxidants, such as butylated hydroxyltoluene (BHT) or butylated hydroxyanisole (BHA). ${ }^{4}$ Phenolic compounds, such as eugenol, are some of the most efficient antioxidants found in essential oils, for instance, various essential oils have shown antioxidant properties owing to presence and high content of eugenol in these volatile oils. ${ }^{5}$ Eugenol (2-methoxy-4-(2propenyl) phenol), yellow oily liquid, is the most abundant component of clove essential oils and exhibits lipic peroxidation induced by reactive oxidase system (ROS) due to its radical scavenging properties. ${ }^{1,4,6}$ The apparent drawbacks of eugenol are its volatile nature and low water solubility; therefore, encapsulation of eugenol is often desired to increase its efficiency and long-term shelf life. ${ }^{7}$

Cyclodextrins (CD), cyclic oligosaccharides having truncated-shape molecular structure, which are capable of forming noncovalent host-guest inclusion complexes (CD/IC) due to their hydrophobic cavity, are often used to encapsulate food/ cosmetic additives, such as flavors/fragrances. ${ }^{8}$ For instance, inclusion complexation of essential oils with $\mathrm{CD}$ provides enhanced water solubility, stabilization against degradation and oxidation (increase the shelf life), controlled and/or delayed release; moreover, it helps to preserve these volatile and hydrophobic molecules. ${ }^{7-9}$ Electrospinning has shown to be one of the promising encapsulation method in which variety of the functional additives for drugs and foods are encapsulated in the nanofiber matrix..$^{10-14}$ In general, electrospinning is a room temperature fiber forming process from polymeric solutions. ${ }^{13}$ In our recent studies, it was shown that the encapsulation efficiency of the volatile active agents, such as menthol, ${ }^{15}$ vanillin, ${ }^{16}$ eugenol, ${ }^{17}$ geraniol, ${ }^{18}$ and allyl isothiocyanate, ${ }^{19}$ was quite ineffective when cyclodextrin inclusion complexes (CD/

Received: September 17, 2017

Revised: November 29, 2017

Accepted: December 18, 2017

Published: December 18, 2017 
IC) were not used. ${ }^{15-19}$ Our studies have shown that high amount of such volatile additives can be effectively preserved by $\mathrm{CD} / \mathrm{IC}$ in the electrospun polymeric nanofibrous matrix. ${ }^{15-19}$ One challenge is to encapsulate higher weight percent (\%) of the additives in the polymeric nanofibrous matrix in the case of encapsulation of $\mathrm{CD} / \mathrm{IC}$ systems. ${ }^{15-20}$ Because, when polymers are used as carrier matrix, the weight $\%$ of the additives in $\mathrm{CD} / \mathrm{IC}$ systems is often limited in the nanofibrous matrix (up to $5 \%(\mathrm{w} / \mathrm{w})$ ), with respect to polymer matrix). This is because the electrospinning of uniform nanofibers is not quite achievable from polymeric solution incorporating higher loading of $\mathrm{CD} / \mathrm{IC}$ (for example, $50 \%$ (w/ w) loading of $\mathrm{CD} / \mathrm{IC}$ corresponds to $\sim 5 \%(\mathrm{w} / \mathrm{w})$ additive when 1:1 molar ratio for guest:CD is considered). So, in our recent studies, ${ }^{21-24}$ nanofibrous webs encapsulating much higher amount of active agents (loading of $\sim 10 \%(\mathrm{w} / \mathrm{w})$ or more with respect to fibrous $\mathrm{CD}$ matrix) were produced from only $\mathrm{CD} / \mathrm{IC}$ systems by polymer-free electrospinning where a fiber forming polymeric matrix was not used for the electrospinning.

In this study, highly concentrated aqueous $\mathrm{CD}$ solutions $(160 \%(\mathrm{w} / \mathrm{v}))$ were prepared and then inclusion complexes between eugenol (guest molecule) and cyclodextrins (as host molecules) were obtained as 1:1 molar ratio of CD:eugenol. Then, these eugenol/CD/IC solutions were successfully transformed into nanofibrous webs (eugenol/CD/IC-NW) by electrospinning technique. The size/shape of $\mathrm{CD}$ cavity is an important parameter to form an effective inclusion complexation, so, three $\mathrm{CD}$ derivatives (HP- $\beta$ - $\mathrm{CD}, \mathrm{HP}-\gamma-\mathrm{CD}$, and $\mathrm{M}-\beta-\mathrm{CD}$ ) were used to form inclusion complexation with eugenol. The resulting electrospun eugenol/CD/IC-NW samples have shown self-standing and flexible character in the form of a mat/web. The morphological (by SEM) and structural (by ${ }^{1} \mathrm{H}$ NMR, XRD, FTIR) analyses, and thermal properties (by TGA) of eugenol/CD/IC-NW were evaluated. It was observed that, eugenol/CD/IC-NW display quite fastdissolving character in water along with high water solubility of eugenol. The thermal property of eugenol is also improved by inclusion complexation in eugenol/CD/IC-NW, additionally, eugenol/CD/IC-NW exhibit effective antioxidant activities.

\section{MATERIALS AND METHODS}

Materials. The hydroxypropyl-beta-cyclodextrin (HP- $\beta$-CD) (DS: $\sim 0.6$, CavasolW7 HP Pharma), hydroxypropyl-gamma-cyclodextrin (HP- $\gamma$-CD) (DS: 0.6, CavasolW8 HP), and methyl-beta-cyclodextrin (M- $\beta$-CD) (DS: 1.6-1.9 CavasolW7M Pharma) were donated by Wacker Chemie AG. Eugenol (99\% purity, SigmaAldrich), deuterated dimethyl sulfoxide (d6-DMSO, 99.8\%, Merck), methanol (extra pure, Sigma-Aldrich), potassium bromide ( $\mathrm{KBr}, 99 \%$, FTIR grade, Sigma-Aldrich), 2,2-diphenyl-1-picrylhydrazyl (DPPH, Sigma-Aldrich) were obtained commercially and used as-received. The water used was from Millipore Milli-Q ultrapure water system.

Preparation of Eugenol/CD/IC Solutions and Electrospinning. Highly concentrated aqueous solutions of eugenol/cyclodextrin inclusion complex (eugenol/CD/IC) were prepared since high solution viscosity is needed for electrospinning of bead-free and uniform fibers. Typically, fiber forming polymeric matrix is used to facilitate the electrospinning process in which long polymer chains sustain the electrospinning jet without break. Nevertheless, using very high concentration of $\mathrm{CD} / \mathrm{IC}$ solutions makes it possible to perform electrospinning of eugenol/CD/IC-NW without the need of polymeric matrix. Each $\mathrm{CD}$ type (HP- $\beta$ - CD, HP- $\gamma$ - $\mathrm{CD}$ and M- $\beta$ $\mathrm{CD})$ having $160 \%(\mathrm{w} / \mathrm{v}$, with respect to solvent; water) concentration was dissolved in water and then certain amount of eugenol was added to have 1:1 molar ratio of $\mathrm{CD}$ :eugenol for each $\mathrm{CD} / \mathrm{IC}$ solution. Each of the $\mathrm{CD} / \mathrm{IC}$ system was stirred overnight at room temperature (RT) to obtain three separate eugenol/CD/IC aqueous solutions (eugenol/ $\mathrm{HP}-\beta$-CD/IC, eugenol/HP- $\gamma$-CD/IC, and eugenol/M- $\beta$-CD/IC). Then, electrospinning of these eugenol/CD/IC aqueous solutions were performed separately in order to produce nanofibrous webs (eugenol/CD/IC-NW). The electrospinning process parameters were applied in the range of $15-20 \mathrm{kV}$ applied voltage, $0.5 \mathrm{~mL} / \mathrm{h}$ feed rate, $10-12 \mathrm{~cm}$ collection distance. Each of eugenol/CD/IC aqueous solution was placed in a syringe having a metallic needle of $0.4 \mathrm{~mm}$ inner diameter and horizontally positioned on a syringe pump (KD Scientific, USA). For the electrospinning of eugenol/CD/IC fibers, HV power supply (Matsusada Precision Inc., Japan) was used and the nanofibrous webs were collected onto aluminum foil covering the grounded static metal collector. During the electrospinning process, the temperature and the relative humidity were recorded as $25^{\circ} \mathrm{C}$ and $18 \%$, respectively. The electrospun nanofibrous webs were detached from $\mathrm{Al}$ foil and these free-standing eugenol/CD/IC-NW samples were dried at $\mathrm{RT}$ and then kept in refrigerator $\left(\right.$ at $4{ }^{\circ} \mathrm{C}$ ) for further characterization.

Measurements and Characterization. The viscosity of eugenol/CD/IC aqueous solutions was measured using cone/plate rheometer (CP 20-2 spindle type, Anton Paar Physica MCR 301) under constant shear rate of $100 \mathrm{~s}^{-1}$ at $23{ }^{\circ} \mathrm{C}$. The Inolab pH/Cond 720-WTW was used to determine the eugenol/CD/IC solution conductivity. The morphology of eugenol/CD/IC-NW was analyzed by scanning electron microscopy (SEM, FEI-Quanta 200 FEG). The sputtering of $\mathrm{Au} / \mathrm{Pd}$ (PECS-682) was applied to nanofibrous webs after placing onto SEM stub to eliminate the charging problem during SEM imaging. The average fiber diameter (AFD) and fiber diameter distribution of the samples were determined from various SEM images recorded at different location of the samples and $\sim 100$ fibers were counted.

For proton nuclear magnetic resonance $\left({ }^{1} \mathrm{H}\right.$ NMR) analysis, 20 $\mathrm{mg} / \mathrm{mL}$ of each eugenol/CD/IC-NW was dissolved in d6-DMSO. The $400 \mathrm{MHz}$ (Bruker DPX-400) type of ${ }^{1} \mathrm{H}$ NMR was used to record the spectra and integration of the chemical shifts $(\delta)$ was performed using Mestrenova software. The thermal stability of pure eugenol and eugenol/CD/IC-NW samples was studied by thermogravimetric analyzer (TGA, TA Q500, USA). TGA measurements were performed under nitrogen atmosphere from 25 to $500{ }^{\circ} \mathrm{C}$ with the heating rate of $20^{\circ} \mathrm{C} / \mathrm{min}$. Fourier transform infrared spectrometer (FTIR) (Bruker-VERTEX70) was used to record the infrared spectra (4000-400 cm $\mathrm{cm}^{-1}$; 64 scans; $4 \mathrm{~cm}^{-1}$ resolution) of samples. Before measurements, samples were turned into pellets by mixing with potassium bromide $(\mathrm{KBr})$ and pressed under pressure. X-ray diffraction (XRD) (PANalytical X'Pert powder diffractometer, $\mathrm{Cu}$ $\mathrm{K} \alpha$ ) was used to study the crystallinity of the samples.

Phase-Solubility Study. The Higuchi and Connors method ${ }^{25}$ was applied for the phase solubility analyses of the $\mathrm{CD}$ :eugenol systems (eugenol/HP- $\beta$-CD/IC, eugenol/HP- $\gamma$-CD/IC, and eugenol/M- $\beta$-CD/IC). For each $\mathrm{CD}$ :eugenol system, each $\mathrm{CD}$ solution $(10 \mathrm{~mL})$ was prepared ranging from 0 to $60 \mathrm{mM}$ concentration. After the addition of excess amount of eugenol, each CD:eugenol solution was kept stirred for $48 \mathrm{~h}$ at RT for equilibrium and then it was filtered using $0.45 \mu \mathrm{m}$ filter membrane to filter the undissolved eugenol. The $\mathrm{CD}$ :eugenol systems were diluted by a definite ratio and eugenol concentration in proportion to increasing $\mathrm{CD}$ concentration was determined by UV/vis Spectrophotometer (Carry 100) at $282 \mathrm{~nm}$ for eugenol. The phase solubility diagrams were plotted for the concentration of eugenol $\left(\mathrm{mM}^{-1}\right)$ versus concentration of $\mathrm{CD}$ $\left(\mathrm{mM}^{-1}\right)$. The average values were determined form three measurements. Moreover, the apparent stability constants $\left(K_{s}\right)$ for eugenol/ $\mathrm{CD} / \mathrm{IC}$ systems were calculated from the phase solubility diagram by using the below equation where $S_{0}$ is the intrinsic solubility of active agents:

$$
K_{\mathrm{s}}=\text { slope } / S_{0}(1-\text { slope })
$$

Eugenol $(\sim 15 \mathrm{mM})$ has quite limited solubility in water. ${ }^{26}$ To visualize the water solubility of eugenol/CD/IC-NW and eugenol, water was added to eugenol/CD/IC-NW $(3 \times 2 \mathrm{~cm}$ web $)$ and pure 

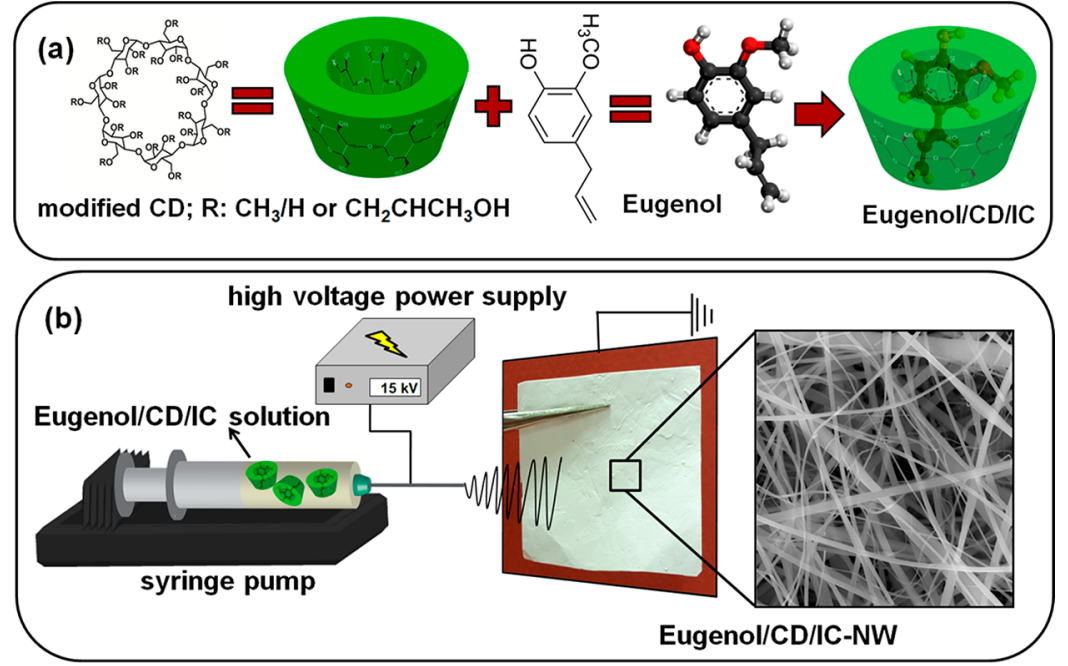

Figure 1. (a) Chemical structure of modified CD, eugenol, and schematic representation of IC formation between CD and eugenol. (b) Schematic representation of the electrospinning of eugenol/CD/IC-NW.
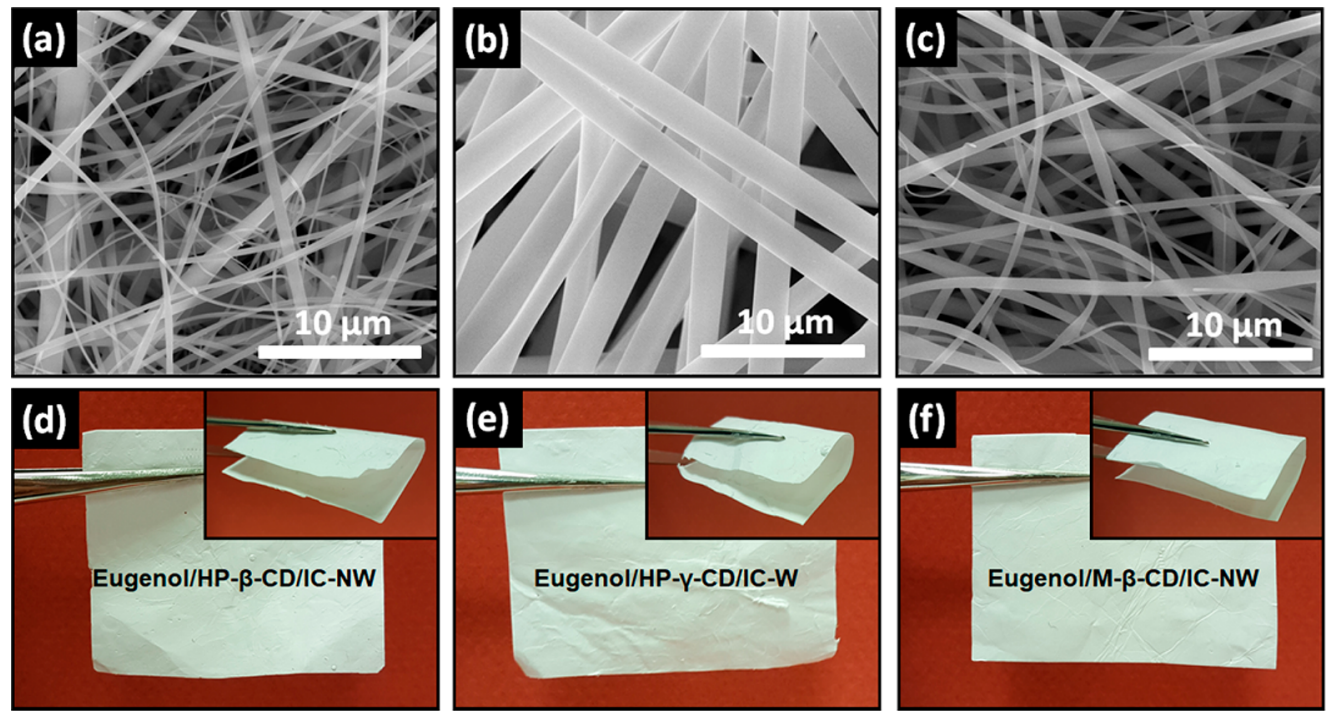

Figure 2. Representative SEM images and the photographs of electrospun (a-d) eugenol/HP- $\beta$-CD/IC-NW, (b-e) eugenol/HP- $\gamma$-CD/IC-W, (c-f) eugenol/M- $\beta$-CD/IC-NW.

eugenol (having the same amount within the nanofibrous webs) in Petri dishes and then both the photographs and videos were recorded (Supporting Information).

Antioxidant Activities of Eugenol/CD/IC-NW. The DPPH radical scavenging method was applied for the antioxidant activity tests. Here, the DPPH solution $(75 \mu \mathrm{M})$ was prepared freshly in methanol and $2.3 \mathrm{~mL}$ of this fresh DPPH solution was mixed with 200 $\mu \mathrm{L}$ of aqueous solutions of eugenol/CD/IC-NW and eugenol. The concentration dependent antioxidant tests of eugenol/CD/IC-NW were performed at $3-1600 \mu \mathrm{g} / \mathrm{mL}$ web concentration ranges. The pure eugenol concentration was calculated from the initial eugenol content which was used for the highest concentrated eugenol/CD/ IC-NW system $(\sim 10 \%(\mathrm{w} / \mathrm{w}$, with respect to total sample amount); $12.8 \mu \mathrm{g} / \mathrm{mL}$ ). The eugenol/CD/IC-NW webs were dissolved instantly in water, but, pure eugenol had to be filtered to remove the undissolved oily eugenol. After the addition of the aqueous solution samples, antioxidant eugenol reduces the DPPH molecules which leads to disappearance of the maximum absorption of DPPH $(517 \mathrm{~nm})$ within incubation time. Here, 50\% inhibition (IC50) concentrations were also calculated from the graph plotting inhibition percentage versus sample concentration. Afterward the time dependent reduction of DPPH was recorded by UV/vis Spectrophotometer.
The experiments were performed in triplicate for the highest nanofibrous web concentration $(1600 \mu \mathrm{g} / \mathrm{mL})$ used for the previous experiment. The below eq (eq 2) was used to determine the DPPH radical scavenging efficiency of the samples in terms of inhibition percentage;

$$
\begin{aligned}
& \text { DPPH radical scavenging }(\%) \\
& \quad=((\text { Blank } \mathrm{AB}-\text { Sample } \mathrm{AB}) / \text { Blank } \mathrm{AB}) \times 100
\end{aligned}
$$

Two-way ANOVA and Tukeỳs multiple comparison tests were applied for statistical analysis of time dependent antioxidant tests. Analysis was done by using GraphPad Prism 5 (GraphPad, USA) at a 0.05 level of probability.

\section{RESULTS AND DISCUSSION}

Morphological Analysis of Nanofibrous Webs. The electrospinning of highly concentrated aqueous solutions of cyclodextrin inclusion complexes of eugenol with three different types of modified cyclodextrins (HP- $\beta$-CD, HP- $\gamma$ $\mathrm{CD}$, and $\mathrm{M}-\beta-\mathrm{CD})$ was successfully performed in order to obtain nanofibrous webs (eugenol/CD/IC-NW) (Figure 1). 
Despite the fact that the eugenol/CD/IC-NW were electrospun from nonpolymeric system (only consist of $\mathrm{CD}$ and eugenol), these eugenol/CD/IC-NW samples have selfstanding and flexible characteristics (Figure 2). Table 1

Table 1. Properties of the Electrospinning Solutions and the Resulting Nanofibers

\begin{tabular}{|c|c|c|c|c|}
\hline solutions & $\begin{array}{l}\text { viscosity } \\
(\mathrm{Pa} \cdot \mathrm{s})\end{array}$ & $\begin{array}{c}\text { conductivity } \\
\left(\mu \mathrm{S} \mathrm{cm}^{-1}\right)\end{array}$ & $\begin{array}{l}\text { average fiber } \\
\text { diameter }(\mathrm{nm})\end{array}$ & $\begin{array}{l}\text { fiber diameter } \\
\text { range }(\mathrm{nm})\end{array}$ \\
\hline $\begin{array}{c}\text { eugenol/HP- } \\
\beta-\mathrm{CD} / \mathrm{IC}\end{array}$ & 0.512 & 19.04 & $600 \pm 375$ & $170-1700$ \\
\hline $\begin{array}{c}\text { eugenol/HP- } \\
\gamma-\mathrm{CD} / \mathrm{IC}\end{array}$ & 0.608 & 9.40 & $1200 \pm 555$ & $350-2430$ \\
\hline $\begin{array}{c}\text { eugenol/M- } \\
\beta \text {-CD/IC }\end{array}$ & 0.466 & 14.50 & $720 \pm 320$ & $220-1430$ \\
\hline
\end{tabular}

summarizes the eugenol/CD/IC solution properties (conductivity and viscosity values) and the resulting average fiber diameter (AFD) of eugenol/CD/IC-NW samples. The representative SEM images of eugenol/CD/IC-NW are given in Figure $2 \mathrm{a}-\mathrm{c}$. As confirmed by SEM imaging, uniform and bead-free nanofibers from all three eugenol/CD/IC systems were produced under the optimized electrospinning conditions/parameters. The AFD values were calculated as $600 \pm$ $375,1200 \pm 555$, and $720 \pm 320 \mathrm{~nm}$ for HP- $\beta$-CD-, HP- $\gamma$ CD-, and $\mathrm{M}-\beta$-CD-based systems, respectively (Table 1 ). As recorded, HP- $\gamma$-CD-based sample has thicker fiber diameter (in the scale of micron $(\mu)$ range), therefore, abbreviation of web (W) was used for the HP- $\gamma$-CD-based sample and nanofibrous web (NW) was used for the HP- $\beta$-CD and M- $\beta$ $\mathrm{CD}$ based samples. Accordingly, the samples were named as eugenol/HP- $\beta$-CD/IC-NW, eugenol/HP- $\gamma$-CD/IC-W, and
eugenol/M- $\beta$-CD/IC-NW. One of the main reasons for thicker fiber diameter for eugenol/HP- $\gamma-\mathrm{CD} / \mathrm{IC}-\mathrm{W}$ is the high solution viscosity of eugenol/HP- $\gamma$-CD/IC which causes less-stretching of the jet during electrospinning. ${ }^{27}$ Moreover, the eugenol/HP- $\gamma$-CD/IC system has also less solution conductivity when compared to eugenol/HP- $\beta-\mathrm{CD} / \mathrm{IC}$ and eugenol/M- $\beta$-CD/IC solutions, causing less-stretching of the jet as well, so thicker fibers were formed for eugenol/HP- $\gamma$ $\mathrm{CD} / \mathrm{IC}-\mathrm{W}$. Although the AFD values of eugenol/HP- $\beta$-CD/ IC-NW and eugenol/M- $\beta$-CD/IC-NW are not that different from each other, eugenol/HP- $\beta$-CD/IC-NW has slightly thinner fibers compared to eugenol/M- $\beta$-CD/IC-NW. Both viscosity and conductivity values of eugenol/HP- $\beta$-CD/IC solution are relatively higher than eugenol/M- $\beta-\mathrm{CD} / \mathrm{IC}$ solution. Here, the higher conductivity of the eugenol/HP- $\beta$ $\mathrm{CD} / \mathrm{IC}$ solution most probably provides more stretching of the jet during the electrospinning process and therefore slightly thinner fibers were obtained for eugenol/HP- $\beta$-CD/IC$\mathrm{NW}^{28-31}$

Eugenol/CD/IC Molar Ratio. The initial molar ratio of $\mathrm{CD}$ :eugenol solution was adjusted as 1:1 to form the inclusion complexation between eugenol and each CD type. Because of volatile nature of eugenol, it is quite possible that the initial amount of eugenol could not be protected during the electrospinning of eugenol/CD/IC solutions or during the storage of eugenol/CD/IC-NW after electrospinning. For instance, our recent studies showed that volatile active agents, such as menthol ${ }^{15}$ or vanillin ${ }^{16}$ or eugenol, ${ }^{17}$ has readily evaporated and therefore could not be preserved effectively if they were only encapsulated in electrospun polymeric nanofibrous webs without cyclodextrin inclusion complexation.
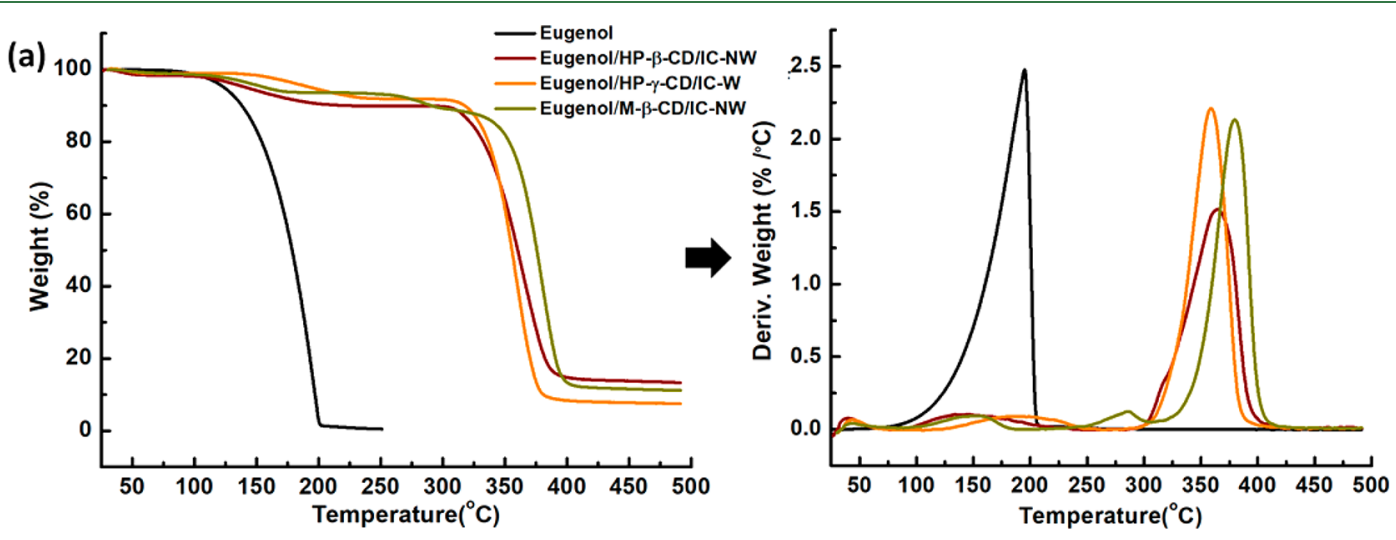

(b)
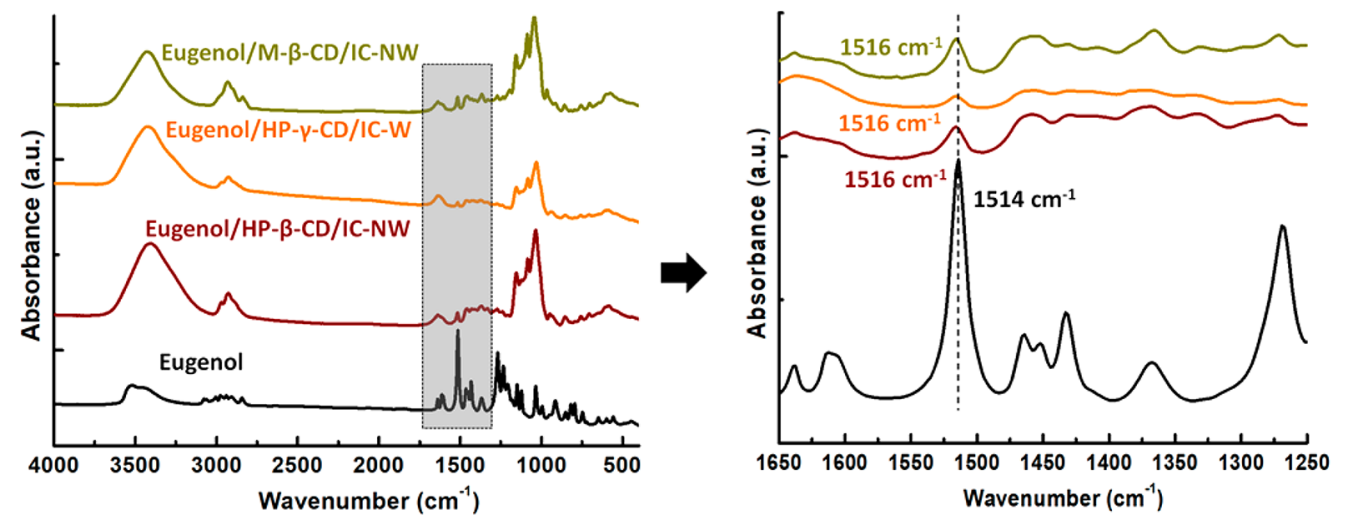

Figure 3. (a) TGA thermograms/derivates and (b) FTIR spectra of eugenol, eugenol/HP- $\beta$-CD/IC-NW, eugenol/HP- $\gamma$-CD/IC-W, and eugenol/ $\mathrm{M}-\beta$-CD/IC-NW. 
Here, the preservation of eugenol in eugenol/CD/IC-NW was quantitatively investigated after electrospinning and storage. For that purpose, ${ }^{1} \mathrm{H}$ NMR was used to further prove the presence and the amount of eugenol in the electrospun nanofibrous matrix. That is, the recorded ${ }^{1} \mathrm{H}$ NMR spectra were used to calculate the molar ratio between $\mathrm{CD}$ (HP- $\beta-\mathrm{CD}$, $\mathrm{HP}-\gamma-\mathrm{CD}$, and M- $\beta$-CD) and eugenol in eugenol/CD/IC-NW samples (Figure S1). The integration of the characteristic peaks belonging to HP- $\beta$-CD (1.00 ppm), HP- $\gamma$-CD (1.00 $\mathrm{ppm}), \mathrm{M}-\beta-\mathrm{CD}(3.5-3.75 \mathrm{ppm})$, and eugenol (6.5 ppm) was used to determine the molar ratio of CD:eugenol for each eugenol/CD/IC-NW sample. Depending on types of CD used, the calculated molar ratio of $\mathrm{CD}$ :eugenol was different for each eugenol/CD/IC-NW sample (Table S1). According to the ${ }^{1} \mathrm{H}$ NMR data, the molar ratio of $\mathrm{CD}$ :eugenol was calculated as 1.00:0.69, 1.00:0.66, and 1.00:0.94 for eugenol/HP- $\beta$-CD/IC$\mathrm{NW}$, eugenol/HP- $\gamma$-CD/IC-W, and eugenol/M- $\beta$-CD/IC$\mathrm{NW}$, respectively. The results indicated that the molar ratio values in eugenol/CD/IC-NW samples are slightly lower compared to their initial ratio (CD:eugenol; $1: 1)$. This is most probably originated from the volatile nature of the eugenol where evaporation of some amount of eugenol took place either during the electrospinning or storage of the samples. Even so, it was seen that significant amount of eugenol was preserved in eugenol/CD/IC-NW and only in the range of $\sim 5-30 \%$ of eugenol was lost from eugenol/CD/IC-NW (Table S1). The results revealed that the type of $\mathrm{CD}$ is important as concluded in our previous studies. ${ }^{21-24}$ Here, M$\beta$-CD was found to be most effective CD type to preserve the eugenol for the electrospun eugenol/M- $\beta$-CD/IC-NW $(\sim 95 \%)$ when compared to eugenol/HP- $\beta$-CD/IC-NW ( $\sim 1 \%)$ and eugenol/HP- $\gamma$-CD/IC-W $(\sim 68 \%)$.

Thermal Analyses of Eugenol/CD/IC-NW. The TGA was used in order to examine the thermal stability of eugenol in eugenol/CD/IC-NW samples. For a comparison study, pure eugenol was also studied. The TGA thermograms of pure eugenol and eugenol/CD/IC-NW samples were depicted in Figure 3a. Eugenol has a volatile nature where the evaporation of pure eugenol was recorded between 60 and $250{ }^{\circ} \mathrm{C}$. For eugenol/CD/IC-NW, three stages of weight loss were observed for eugenol/HP- $\beta$-CD/IC-NW and eugenol/HP- $\gamma$ $\mathrm{CD} / \mathrm{IC}-\mathrm{W}$. The first weight loss below $100{ }^{\circ} \mathrm{C}$ belongs to water evaporation and the third weight loss above $300{ }^{\circ} \mathrm{C}$ belongs to main degradation of $\mathrm{CD}$ for eugenol/CD/IC-NW samples. The second weight losses due to evaporation of eugenol between 90 and $250{ }^{\circ} \mathrm{C}$ and $125-250{ }^{\circ} \mathrm{C}$ were recorded for eugenol/HP- $\beta$-CD/IC-NW and eugenol/HP- $\gamma$ $\mathrm{CD} / \mathrm{IC}-\mathrm{W}$, respectively. The shifting of thermal evaporation of eugenol to higher temperature clearly reveals the inclusion complexation state between host CD (HP- $\beta$-CD and HP- $\gamma$ $\mathrm{CD}$ ) and eugenol for these samples. On the other hand, twostep weight loss was observed at $90-190{ }^{\circ} \mathrm{C}$ and $190-310^{\circ} \mathrm{C}$ for eugenol evaporation in the case of Eugenol/M- $\beta$-CD/ICNW sample. This may indicate the presence of two different types of complexation between $\mathrm{M}-\beta-\mathrm{CD}$ and eugenol. The thermal evaporation of eugenol shifted to higher temperature for eugenol/M- $\beta$-CD/IC-NW when compared to eugenol/HP$\beta$-CD/IC-NW and eugenol/HP- $\gamma$-CD/IC-W (Figure 3a). In our previous studies, ${ }^{22-24}$ similar results were also recorded for the inclusion complexation between $\mathrm{M}-\beta-\mathrm{CD}$ and some other active compounds (i.e., vanillin, limonene, linalool), and the possible explanation is that the methyl groups of $\mathrm{M}-\beta-\mathrm{CD}$ might increase the hydrophobic interaction and provide higher stability to these $\mathrm{CD} / \mathrm{IC}$ systems. In addition, while water \% content $(w / w$, with respect to the sample) observed up to 100 ${ }^{\circ} \mathrm{C}$, is in the range of $2.5-5.8 \%$ for pure modified $\mathrm{CD}$ nanofibers (as it was recorded in our previous study ${ }^{31}$ ), here, water $\%$ content was calculated in the range of $0.9-1.4 \%$ for eugenol/CD/IC-NW samples. This distinct decrease in water content is because of the replacement of water molecules by active agent molecules in the $\mathrm{CD}$ cavity during inclusion complex formation. From the TGA data, the amount of eugenol in eugenol/CD/IC-NW was also calculated and summarized in Table S1. The total amount of eugenol in eugenol/HP- $\beta$-CD/IC-NW, eugenol/HP- $\gamma$-CD/IC-W, and eugenol/M- $\beta$-CD/IC-NW samples was determined as 8.4 , 7.0 , and $10.1 \%(\mathrm{w} / \mathrm{w}$, according to total sample amount), respectively. The molar ratios of eugenol were calculated based on TGA results and shown in Table S1. As it is seen, the molar ratios calculated from TGA results were not exactly matching with the results obtained from ${ }^{1} \mathrm{H} \mathrm{NMR}$, however, the molar ratio values were quite close to each other. From the TGA data, eugenol/M- $\beta$-CD/IC-NW has shown the highest encapsulation efficiency which correlates with the ${ }^{1} \mathrm{H}$ NMR findings. The efficient complexation of $\mathrm{M}-\beta-\mathrm{CD}$ with eugenol in eugenol/CD/IC-NW sample might be originated from the modifications variations between the $\mathrm{CD}$ types. Unlike $\mathrm{M}-\beta$ $\mathrm{CD}$, the hydroxypropyl group of HP- $\beta$-CD and HP- $\gamma-\mathrm{CD}$ might create steric hindrance which can prevent the efficient inclusion of eugenol within the CD cavity. ${ }^{32,33}$ Additionally, the substitution of hydroxypropyl moiety can cause axial elongation at the $\mathrm{CD}$ torus, ${ }^{34}$ consequently, $\mathrm{M}-\beta$ - $\mathrm{CD}$ cavity might be a more favorable for eugenol compared to HP- $\beta$-CD and HP- $\gamma-\mathrm{CD}$. Briefly, TGA calculations confirmed that substantial part of eugenol $(\sim 80.0, \sim 75$, and $\sim 92 \%$ for eugenol/HP- $\beta$-CD/IC-NW, eugenol/HP- $\gamma$-CD/IC-W, and eugenol/M- $\beta$-CD/IC-NW, respectively) was preserved during the electrospinning processes and/or storage of the eugenol/ CD/IC-NW samples. Additionally, eugenol/CD/IC-NW samples could preserve much higher eugenol content compared to polymeric nanofiber matrix. For instance, in our previous study, eugenol could not be protected at all in poly(vinyl alcohol) (PVA) electrospun nanofibers without $\mathrm{CD} / \mathrm{IC}^{17}$

Structural Characterization of Eugenol/CD/IC-NW. The FTIR analyses were performed to confirm the presence of eugenol and its inclusion complexation for eugenol/CD/ICNW samples. Figure $3 \mathrm{~b}$ displays the FTIR spectra of the pure eugenol and samples of eugenol/HP- $\beta$-CD/IC-NW, eugenol/ $\mathrm{HP}-\gamma$-CD/IC-W, and eugenol/M- $\beta$-CD/IC-NW. Due to presence of $\mathrm{CD}$, the eugenol/CD/IC-NW samples have major absorptions at around 1020-1070, 1150, 1638, 2925, and $3401 \mathrm{~cm}^{-1}$ correspond to the coupled $\mathrm{C}-\mathrm{C} / \mathrm{C}-\mathrm{O}$ stretching vibrations, antisymmetric stretching vibration of the $\mathrm{C}-\mathrm{O}-\mathrm{C}$ glycosidic bridge, $\mathrm{H}-\mathrm{OH}$ bending, $\mathrm{C}-\mathrm{H}$ stretching, and $\mathrm{O}-\mathrm{H}$ stretching, respectively. ${ }^{24}$ The signature peaks of eugenol at the range of $1250-1650 \mathrm{~cm}^{-1}$ are corresponding to $\mathrm{C}=\mathrm{C}$ stretching and $\mathrm{C}-\mathrm{H}$ bending of the alkene/aromatic groups of eugenol (Figure $3 \mathrm{~b}$ ). ${ }^{17}$ Since most of the characteristic peaks of CD overlap with eugenol peaks, this makes it difficult to differentiate each component in the eugenol/CD/IC-NW samples. Yet, while the characteristic peak of eugenol at $\sim 1516 \mathrm{~cm}^{-1}$ originates from the $\mathrm{C}=\mathrm{C}$ stretching was observed for all eugenol/CD/IC-NW samples (Figure $3 \mathrm{~b}$ ), this peak is absent in the FTIR spectra of pure CD samples (Figure S2). So, the peak observed at $\sim 1516 \mathrm{~cm}^{-1}$ for 
(a)

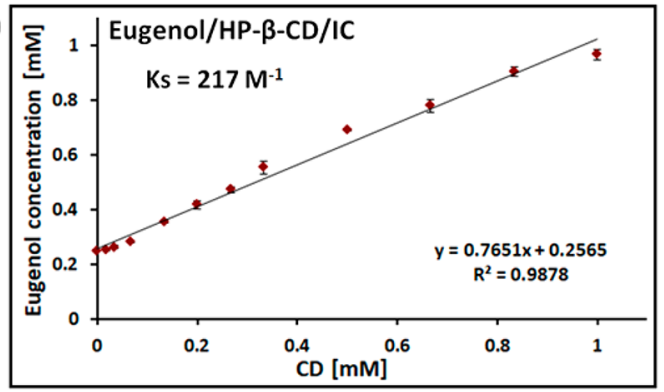

(b)

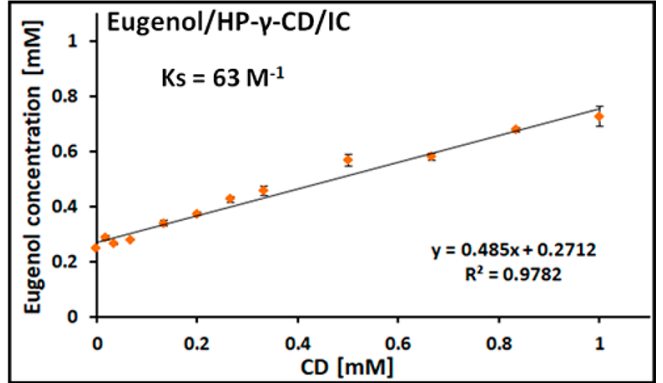

(c)

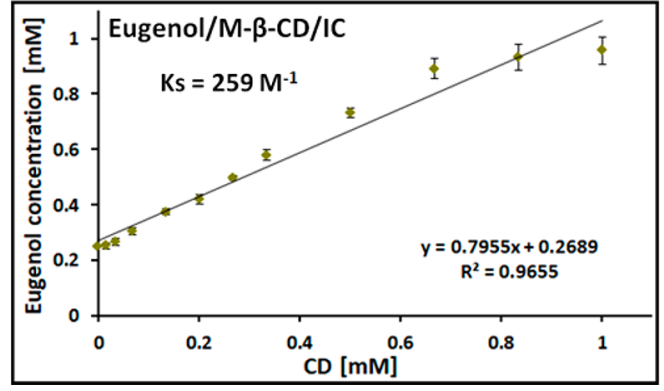

Figure 4. Phase solubility diagrams of (a) eugenol/HP- $\beta$-CD/IC, (b) eugenol/HP- $\gamma$-CD/IC, and (c) eugenol/M- $\beta$-CD/IC systems.

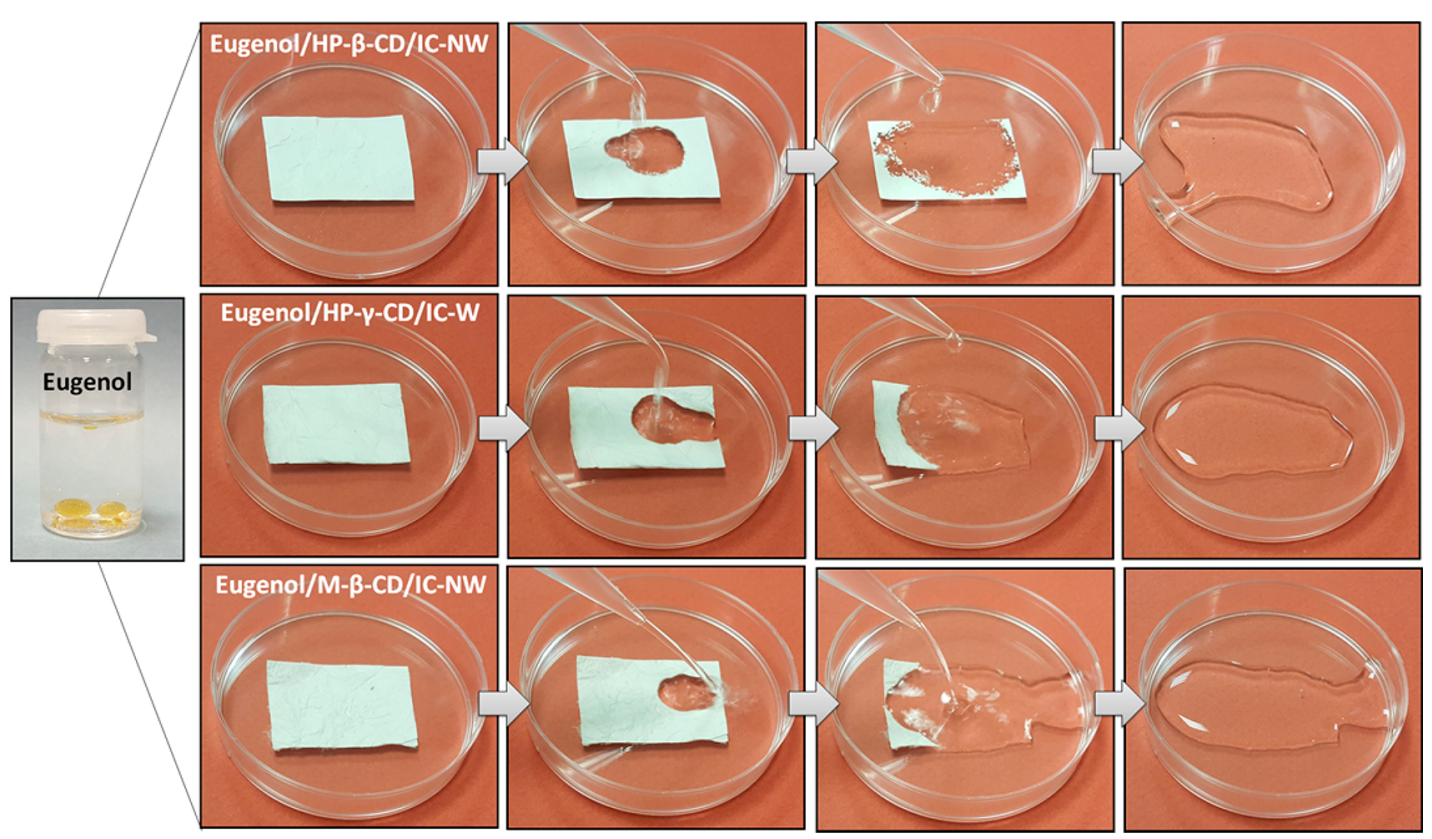

Figure 5. Presentation of the solubility behavior of eugenol, eugenol/H-P- $\beta$ CD/IC-NW, eugenol/HP- $\gamma-\mathrm{CD} / \mathrm{IC}-\mathrm{W}$, and eugenol/M- $\beta$-CD/ICNW. The pictures are taken after a very few seconds of water exposure (see Movie S1).

eugenol/CD/IC-NW samples indicated the presence of eugenol in these samples. In the case of inclusion complexation, it is also anticipated that certain interactions between $\mathrm{CD}$ and guest molecule cause some peak shifts at the FTIR spectra of the $\mathrm{CD} / \mathrm{IC} .^{24}$ Here, it was observed that, the characteristic peak of pure eugenol at $1514 \mathrm{~cm}^{-1}$ was shifted to $1516 \mathrm{~cm}^{-1}$ which further proved the inclusion complex formation between eugenol and CD in eugenol/CD/IC-NW samples. Here, X-ray diffraction (XRD) was also used to confirm the inclusion complex formation between $\mathrm{CD}$ molecules and eugenol. The modified CD, HP- $\beta$-CD, HP- $\gamma$ $\mathrm{CD}$, and $\mathrm{M}-\beta-\mathrm{CD}$, are known with their amorphous structure. ${ }^{31}$ The liquid state of eugenol at RT prevented the $\mathrm{XRD}$ measurement for pure compound; even so, eugenol/CD/
IC-NW demonstrated amorphous structure as well. In addition there is no crystal formation of eugenol in Eugenol/CD/ICNW confirming the inclusion complexation (Figure S3).

Eugenol/CD Phase Solubility Tests. Eugenol is used in food products, but it can suffer from poor water solubility which sometimes limits its application. In this study, we have performed phase solubility tests to indicate the apparent solubility enhancement of eugenol as a function of increasing CD concentration. Higuchi and Connors demonstrated that, ${ }^{25}$ there are different types of phase solubility diagrams depending on the stoichiometric variations between $\mathrm{CD}$ and guest molecules during the inclusion complexation. In our case, phase solubility diagrams of eugenol/CD/IC display $A_{L}$ type pattern. There is a linear increase in the solubility of eugenol 

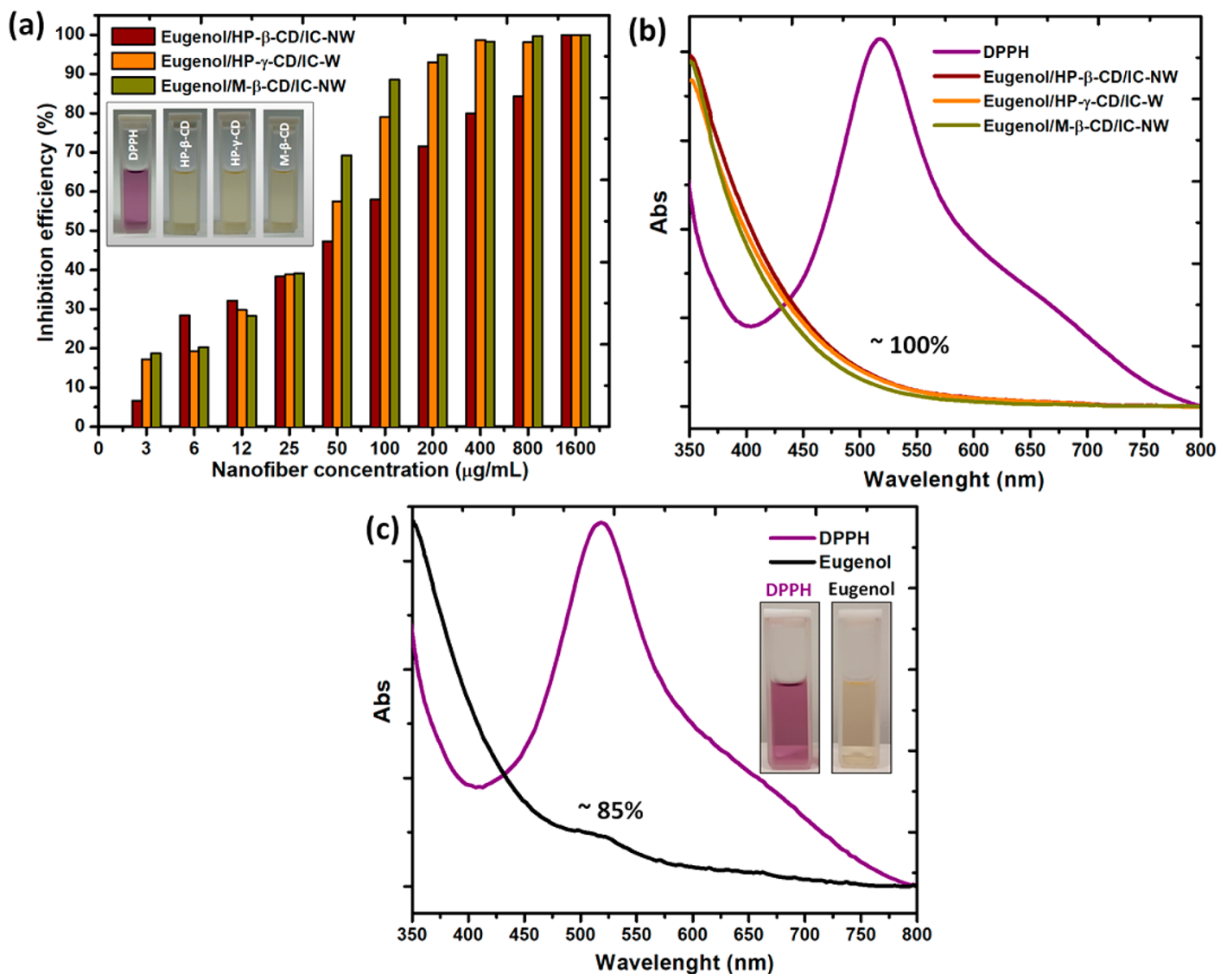

Figure 6. Sample concentration dependent antioxidant test graphs and the resulting DPPH solution photographs of (a) eugenol/HP- $\beta$-CD/IC$\mathrm{NW}$, eugenol/HP- $\gamma-\mathrm{CD} / \mathrm{IC}-\mathrm{W}$, and eugenol/M- $\beta$-CD/IC-NW. (b) UV-vis absorption graphs of antioxidant tests taking for the highest concentrations for eugenol/CD/IC-NW $(1600 \mu \mathrm{g} / \mathrm{mL})$. (c) UV-vis absorption graphs and the DPPH solution photographs eugenol (12.8 $\mu \mathrm{g} /$ $\mathrm{mL}$ ) after radical scavenging tests.

with the increase in $\mathrm{CD}$ concentration, and this also reveals the 1:1 molar ratio inclusion complex formation between eugenol and $\mathrm{CD}$ (Figure 4). Here, HP- $\beta$-CD and M- $\beta$-CD provided relatively higher increment at the solubility of eugenol compared to HP- $\gamma$-CD by a linear manner for the chosen $\mathrm{CD}$ concentration range. Besides, stability constant $\left(K_{\mathrm{s}}\right)$ values belong to $\mathrm{CD} / \mathrm{IC}$ were calculated according to eq 1 by using phase solubility diagrams. $K_{\mathrm{s}}$ values were calculated as 217,63 , and $259 \mathrm{M}^{-1}$ for eugenol/HP- $\beta$-CD/IC, eugenol/HP- $\gamma$-CD/ IC, and eugenol/M- $\beta$-CD/IC, respectively. Principally, $K_{\mathrm{s}}$ represent the binding strength between guest molecules and $\mathrm{CD}^{7,34}$ and based on our calculations, it was concluded that, HP- $\beta$-CD and M- $\beta$-CD can form more stable complexes with eugenol compared to HP- $\gamma$-CD. This might be originated from the bigger cavity size of HP- $\gamma$-CD which is not as successful as $\mathrm{HP}-\beta-\mathrm{CD}$ and $\mathrm{M}-\beta-\mathrm{CD}$ for stabilizing the interaction with eugenol in the aqueous environment. Hence, this leads to encapsulation of less amount of eugenol in HP- $\gamma-\mathrm{CD}$ and so resulted in lower water solubility when compared to HP- $\beta$-CD and M- $\beta$-CD. When eugenol/HP- $\beta$-CD/IC and eugenol/M- $\beta$ $\mathrm{CD} / \mathrm{IC}$ are compared to each other, $\mathrm{M}-\beta$-CD-based system $\left(259 \mathrm{M}^{-1}\right)$ indicates slightly higher stability constant than HP$\beta$-CD system $\left(217 \mathrm{M}^{-1}\right)$. Due to the stronger binding between eugenol and $M-\beta-C D$, higher amount of eugenol was also encapsulated in eugenol/M- $\beta$-CD/IC-NW as confirmed by ${ }^{1} \mathrm{H}$ NMR and TGA results.

Eugenol/CD/IC-NW Water Solubility Studies. The water solubility enhancement of the eugenol in eugenol/CD/
IC-NW was evaluated by dissolving them in water. The eugenol/CD/IC-NW samples were dissolved in water immediately upon water contact, whereas the pure eugenol remained undissolved in water even after stirring overnight (Figure 5 and Movie S1). These visualizations revealed that, water-soluble eugenol was obtained in CD/IC-NW as a results of inclusion complex formation. The contribution of high surface area and nanoscale porosity of electrospun nanofibrous webs can also be the reason for the fast-dissolving property of eugenol/CD/IC-NW.

Eugenol/CD/IC-NW Antioxidant Activity Tests. Reactive oxygen species (ROS) and free radicals can cause diseases/ aging or spoiling of foods by lipid peroxidation in biological systems and biomolecules. ${ }^{1,5}$ Essential oils extracted from the natural plants have been broadly used in food, pharmaceutical and cosmetic industries due to their antioxidant properties. The high antioxidant potential of essential oils is derived from a wide range of volatile compounds such as terpenes, terpenoids, phenolic aromatic compounds, and aliphatic components. ${ }^{1,5}$ In a study of Anthony and co-workers, the antioxidant properties of 423 essential oils from 48 different botanical families were evaluated and it was accepted that the essential oil families possess significant antioxidant activities generally due to their content of eugenol. ${ }^{5}$ The related studies demonstrated that the antioxidant capacity of eugenol has been attributed to its phenol groups which can inhibit the lipidic peroxidation induced by ROS. There are various approaches to examine the antioxidant properties of materials. ${ }^{35}$ The use of 


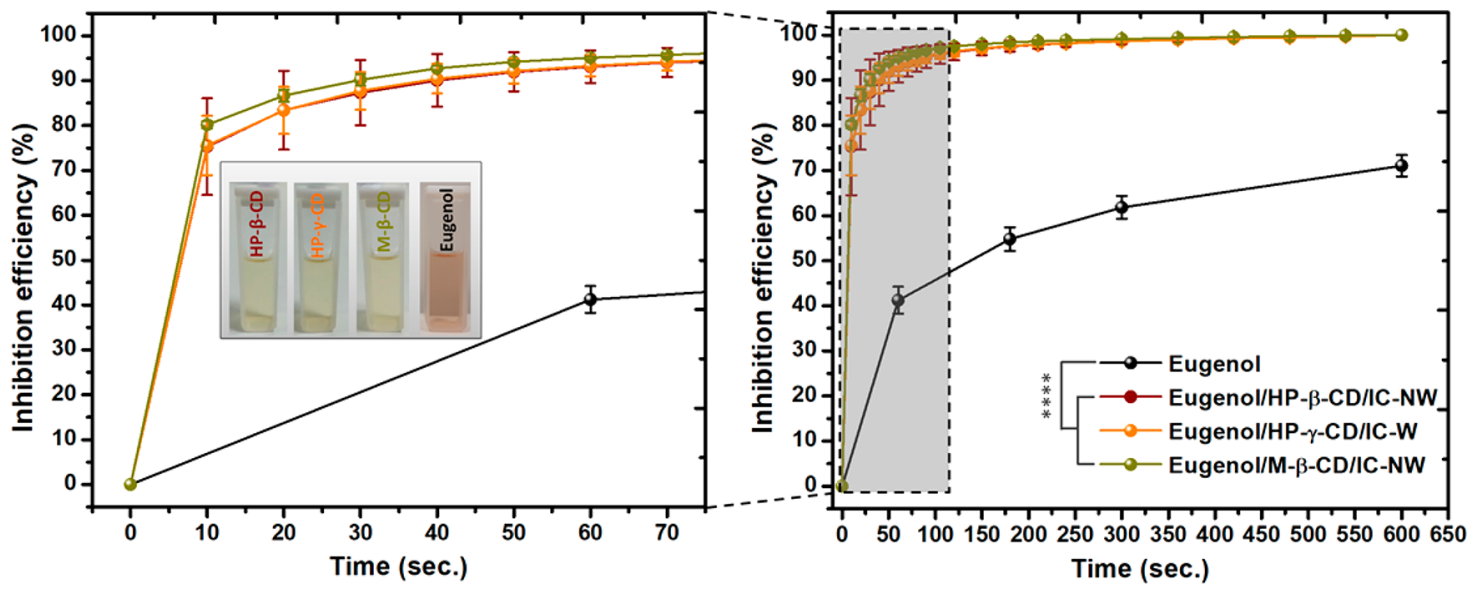

Figure 7. Time dependent antioxidant test graphs and the resulting photographs of eugenol, eugenol/H-P- $\beta \mathrm{CD} / \mathrm{IC}-\mathrm{NW}$, eugenol/HP- $\gamma$-CD/IC$\mathrm{W}$, and eugenol/M- $\beta$-CD/IC-NW (narrowed range and full range, respectively). $n=3$, the error bars; standard deviation, ns: not significant, $*: p \leq$ $0.05, * *: p \leq 0.01, * * *: p \leq 0.001, * * * *: p \leq 0.0001$.

DPPH is a practical method when radical scavenging abilities of components are investigated at room temperature. ${ }^{36,37}$ In this method, DPPH molecule is reduced by a hydrogen donor of free radical scavenging antioxidant. Due to DPPH reduction, the salient absorption band at $517 \mathrm{~nm}$ decreases and the color of the solution totally turns from purple to yellowish at the end of the reaction (Figure 6).

In this study, the concentration dependent antioxidant tests of eugenol/CD/IC-NW were performed at 3-1600 $\mu \mathrm{g} / \mathrm{mL}$ nanoweb concentration ranges. Here, the clear solutions were used for eugenol/CD/IC-NW samples. As a control experiment, pure eugenol was prepared according to highest eugenol/CD/IC-NW amount $(1600 \mu \mathrm{g} / \mathrm{mL})$ and by using the initial eugenol content $(10 \% \mathrm{w} / \mathrm{w}$, with respect to the total sample amount) applied for the preparation of electrospinning solutions. Since the pure eugenol could not enable the preparation of clear and homogeneous solution, the undissolved eugenol was filtered just before the antioxidant experiment. The UV/vis measurements of samples were performed after $30 \mathrm{~min}$ incubation time for all concentrations of eugenol/CD/IC-NW and pure eugenol (Figure 6). Figure 6 a displays the \% DPPH radical scavenging graphs of eugenol/ $\mathrm{CD} / \mathrm{IC}-\mathrm{NW}$ depending on the concentration and the photographs of the ultimate solutions for all systems. As it is expected, DPPH radicals were scavenged in a dose-dependent manner for eugenol/CD/IC-NW and the inhibition efficiency of samples increases by the increasing sample concentrations. For eugenol/CD/IC-NW, the radical scavenging was completed at the highest sample concentrations $(1600 \mu \mathrm{g} / \mathrm{mL})$ after $30 \mathrm{~min}$ incubation time ( $100 \%$ antioxidant activity) (Figure 6a,b). The concentration providing 50\% inhibition (IC50) was calculated for each sample and the results were 62, 40 , and $32 \mu \mathrm{g} / \mathrm{mL}$ for eugenol/HP- $\beta$-CD/IC-NW, eugenol/ $\mathrm{HP}-\gamma$-CD/IC-W, and eugenol/M- $\beta$-CD/IC-NW, respectively. It is clear that the lowest amount of eugenol/M- $\beta$-CD/IC-NW is needed to exhibit the IC50 when compared to eugenol/HP$\beta$-CD/IC-NW and eugenol/HP- $\gamma$-CD/IC-W. The possible reason is the higher eugenol content in eugenol/M- $\beta$-CD/IC$\mathrm{NW}$ compared to eugenol/H-P- $\beta \mathrm{CD} / \mathrm{IC}-\mathrm{NW}$ and eugenol/ $\mathrm{HP}-\gamma$-CD/IC-W samples. Eugenol is a monophenolic compound and despite the availability of one hydrogen on a hydroxyl group, eugenol molecules are able to reduce more than one DPPH molecules, since the carbon in the para- position having more than two hydrogen, enables the donation of second hydrogen by the electron delocalization onto this para-substituted group. ${ }^{38}$ When eugenol makes an inclusion complexation with $\mathrm{CD}$, the phenolic group of eugenol is positioned near to the wider rim of $\mathrm{CD}$ cavity. ${ }^{39}$ Due to this possible positioning of eugenol in $\mathrm{CD}$ cavity, the inclusion complex formation does not hinder the radical scavenging property of eugenol. ${ }^{6}$ Here, the pure eugenol system indicates $85 \%$ antioxidant activity under the same condition with eugenol/CD/IC-NW (100\% antioxidant activity, Figure 6b). The enhanced antioxidant activity for eugenol/CD/IC-NW could be due to the effective complexation of eugenol with $\mathrm{CD}$ which provides stabilization and higher water solubility; hence more amount of eugenol become available for the scavenging process.

To reveal the antioxidant efficiency differences among samples more clearly, time dependent radical scavenging tests were performed from the highest sample concentration which was used for the previous concentration dependent experiment protocols $(1600 \mu \mathrm{g} / \mathrm{mL})$. The graphs of time dependent DPPH scavenging clearly present the differences for antioxidant activity between eugenol and eugenol/CD/IC-NW samples (Figure 7). Despite the same amount of eugenol was used within eugenol/CD/IC-NW, pure eugenol demonstrated much more slow and ineffective antiradical reaction profile compared to eugenol/CD/IC-NW in the given time period. While the radical scavenging reactions were completed by eugenol/CD/IC-NW, pure eugenol could not provide a full scavenging. This is originated from the enhanced water solubility of eugenol gained with inclusion complexation which increases the amount of eugenol could take part in the scavenging process. For eugenol/CD/IC-NW, the graph drawn until $75 \mathrm{~s}$ shows that there is not so much differences between the reaction rates of eugenol/CD/IC-NW depending on the modified $\mathrm{CD}$ types, however, eugenol/M- $\beta$-CD/IC$\mathrm{NW}$ sample show slightly faster reaction than eugenol/H-P$\beta \mathrm{CD} / \mathrm{IC}-\mathrm{NW}$ and eugenol/HP- $\gamma$-CD/IC-W samples. This is most probably due to the its higher eugenol content as confirmed by ${ }^{1} \mathrm{H}$ NMR and TGA measurement (Table S1). Eugenol/HP- $\beta$-CD/IC-NW includes slightly higher amount of eugenol compared to eugenol/HP- $\gamma$-CD/IC-W, even so, they have performed at the similar reaction rate. This radical scavenging profile of eugenol/HP- $\gamma$-CD/IC-W could reflect 
the presence of more relaxed interaction between eugenol and HP- $\gamma$-CD compared to HP- $\beta$-CD due to the bigger cavity size of HP- $\gamma$-CD which might soften hindrance on the oxidation of eugenol and make easier for eugenol to indicate its reduction potential. In brief, superior antioxidant activity was achieved for electrospun eugenol/CD/IC-NW compared to pure eugenol.

To conclude, self-standing and flexible nanofibrous webs were successfully produced from the inclusion complexes (IC) of three modified CD (HP- $\beta-\mathrm{CD}, \mathrm{HP}-\gamma-\mathrm{CD}$, and $\mathrm{M}-\beta-\mathrm{CD})$ and volatile essential oil extraction product (eugenol), via electrospinning technique. The eugenol/CD/IC-NW samples from each $\mathrm{CD}$ type were obtained with bead-free fiber morphology. The presence and the amount of eugenol in the eugenol/CD/IC-NW samples was calculated by ${ }^{1} \mathrm{H}$ NMR studies. It was found out that significant amount of eugenol ( $70-95 \%)$ was preserved in the eugenol/CD/IC-NW owing to inclusion complexation of eugenol with $\mathrm{CD} .{ }^{1} \mathrm{H} \mathrm{NMR}$ results revealed that eugenol/HP- $\beta$-CD/IC-NW, eugenol/HP$\gamma$-CD/IC-W, eugenol/M- $\beta$-CD/IC-NW preserved up to $\sim 71$, $\sim 68$, and $\sim 95 \%$ of initial amount of eugenol, respectively. The structural analyses by FTIR and XRD further reveals the inclusion complexation of eugenol with $\mathrm{CD}$ in eugenol/M- $\beta$ $\mathrm{CD} / \mathrm{IC}-\mathrm{NW}$ samples. TGA measurements also proved the inclusion complex formation between eugenol and CD since the eugenol evaporation took place at significantly higher temperature (up to $310^{\circ} \mathrm{C}$ ) when compared to pure form of eugenol (up to $200{ }^{\circ} \mathrm{C}$ ). Thus, it can be concluded that eugenol/CD/IC-NW samples have shown enhanced thermal stability for eugenol due the inclusion complex formation. The water solubility enhancement provided by inclusion complexation was demonstrated via phase solubility diagrams of eugenol/CD/IC systems. Additionally, it was observed that free-standing eugenol/CD/IC-NW were readily dissolved in water whereas the pure eugenol has obvious poor water solubility. To conclude, both the thermal stability and the water solubility of eugenol are well improved by the inclusion complexation with $\mathrm{CD}$ molecules. Moreover, $\mathrm{M}-\beta-\mathrm{CD}$ tends to form more stable inclusion complexes with eugenol along with higher encapsulation efficiency when compared to HP- $\beta$-CD and HP- $\gamma$-CD. The DPPH radical scavenging tests confirmed the improved radical scavenging property of eugenol/CD/IC$\mathrm{NW}$ compared to the pure state of eugenol owing to its enhanced solubility by inclusion complexation with $\mathrm{CD}$ molecules. Briefly, eugenol was encapsulated into more applicable free-standing and easily handled nanofibrous forms having high eugenol content along with the effective preservation of eugenol. In short, the polymer-free electrospinning (without using fiber forming polymeric matrix) of nanofibrous webs from only $\mathrm{CD} / \mathrm{IC}$ systems provides better encapsulation and much higher loading of active agents. Hence, eugenol/CD/IC-NW or other electrospun CD/IC systems encapsulating antioxidant additives could be quite applicable for food related applications due to their large surface area along with cyclodextrin inclusion complexation providing better antioxidant activity, enhanced water-solubility and high temperature stability.

\section{ASSOCIATED CONTENT}

\section{S Supporting Information}

The Supporting Information is available free of charge on the ACS Publications website at DOI: 10.1021/acs.jafc.7b04312.
${ }^{1} \mathrm{H}$ NMR and TGA calculations indicating the eugenol amount in $\mathrm{CD} / \mathrm{IC}-\mathrm{F}$ in terms of molar ratio (CD:eugenol)/\% (w/w) comparatively with their initial values; ${ }^{1} \mathrm{H}$ NMR spectra of eugenol/HP- $\beta$-CD/IC-NW, eugenol/HP- $\gamma$-CD/IC-W, and eugenol/M- $\beta$-CD/ICNW dissolved in $66-\mathrm{DMSO}$; FTIR spectra of pure $\mathrm{HP}-\beta-\mathrm{CD} / \mathrm{NW}, \mathrm{HP}-\gamma-\mathrm{CD} / \mathrm{NW}$, and $\mathrm{M}-\beta-\mathrm{CD} / \mathrm{NW}$; and XRD patterns of eugenol/HP- $\beta$-CD/IC-NW, eugenol/ $\mathrm{HP}-\gamma$-CD/IC-W, and eugenol/M- $\beta$-CD/IC-NW (PDF) Movie of the water solubility of eugenol/CD/IC-F (AVI)

\section{AUTHOR INFORMATION}

\section{Corresponding Author}

*Telephone: +90-3122908987; E-mail: tamer@unam.bilkent. edu.tr.

ORCID

Tamer Uyar: 0000-0002-3989-4481

Funding

Dr. Uyar acknowledges The Scientific and Technological Research Council of Turkey (TUBITAK)-Turkey (Project \# 213M185) for funding this research. A.C. and Z.I.Y. thank TUBITAK-BIDEB for the PhD scholarship.

\section{Notes}

The authors declare no competing financial interest.

\section{REFERENCES}

(1) Bakkali, F.; Averbeck, S.; Averbeck, D.; Idaomar, M. Biological effects of essential oils-a review. Food Chem. Toxicol. 2008, 46, 446475.

(2) Acosta, S.; Chiralt, A.; Santamarina, P.; Rosello, J.; GonzálezMartínez, C.; Cháfer, M. Antifungal films based on starch-gelatin blend, containing essential oils. Food Hydrocolloids 2016, 61, 233240.

(3) Llana-Ruiz-Cabello, M.; Pichardo, S.; Maisanaba, S.; Puerto, M.; Prieto, A. I.; Gutierrez-Praena, D.; Jos, A.; Camean, A. M. In vitro toxicological evaluation of essential oils and their main compounds used in active food packaging: a review. Food Chem. Toxicol. 2015, 81, 9-27.

(4) Li, K. K.; Yin, S. W.; Yang, X. Q.; Tang, C. H.; Wei, Z. H. Fabrication and characterization of novel antimicrobial films derived from thymol-loaded zein-sodium caseinate (SC) nanoparticles. J. Agric. Food Chem. 2012, 60, 11592-11600.

(5) Anthony, K. P.; Deolu-Sobogun, S. A.; Saleh, M. A. Comprehensive assessment of antioxidant activity of essential oils. J. Food Sci. 2012, 77, C839.

(6) Babaoglu, H. C.; Bayrak, A.; Ozdemir, N.; Ozgun, N. Encapsulation of clove essential oil in hydroxypropyl beta-cyclodextrin for characterization, controlled release, and antioxidant activity. J. Food Process. Preserv. 2017, 41, e13202.

(7) Marques, H. M. C. A review on cyclodextrin encapsulation of essential oils and volatiles. Flavour Fragrance J. 2010, 25, 313-326.

(8) Del Valle, E. M. Cyclodextrins and their uses: a review. Process Biochem. 2004, 39, 1033-1046.

(9) Szente, L.; Szejtli, J. Cyclodextrins as food ingredients. Trends Food Sci. Technol. 2004, 15, 137-142.

(10) Hu, X.; Liu, S.; Zhou, G.; Huang, Y.; Xie, Z.; Jing, X. Electrospinning of polymeric nanofibers for drug delivery applications. J. Controlled Release 2014, 185, 12-21.

(11) Pérez-Mariá, R.; Fabra, M. J.; Lagarón, J. M.; López-Rubio, A. Use of electrospinning for encapsulation. Encapsulation Nanotechnologies; John Wiley \& Sons, 2013.

(12) Noruzi, M. Electrospun nanofibres in agriculture and the food industry: a review. J. Sci. Food Agric. 2016, 96, 4663-4678. 
(13) Electrospun Materials for Tissue Engineering and Biomedical Applications: Research, Design and Commercialization; Uyar, T., Kny, E., Eds.; Elsevier Woodhead Publishing Series in Biomaterials, 2017.

(14) Wen, P.; Wen, Y.; Zong, M.-H.; Linhardt, R. J.; Wu, H. Encapsulation of Bioactive Compound in Electrospun Fibers and Its Potential Application. J. Agric. Food Chem. 2017, 65, 9161-9179.

(15) Uyar, T.; Hacaloglu, J.; Besenbacher, F. Electrospun polyethylene oxide (PEO) nanofibers containing cyclodextrin inclusion complex. J. Nanosci. Nanotechnol. 2011, 11, 3949-3958.

(16) Kayaci, F.; Uyar, T. Encapsulation of vanillin/cyclodextrin inclusion complex in electrospun polyvinyl alcohol (PVA) nanowebs: prolonged shelf-life and high temperature stability of vanillin. Food Chem. 2012, 133, 641-649.

(17) Kayaci, F.; Ertas, Y.; Uyar, T. Enhanced thermal stability of eugenol by cyclodextrin inclusion complex encapsulated in electrospun polymeric nanofibers. J. Agric. Food Chem. 2013, 61, 8156-8165. (18) Kayaci, F.; Sen, H. S.; Durgun, E.; Uyar, T. Functional electrospun polymeric nanofibers incorporating geraniol-cyclodextrin inclusion complexes: High thermal stability and enhanced durability of geraniol. Food Res. Int. 2014, 62, 424-431.

(19) Aytac, Z.; Dogan, S. Y.; Tekinay, T.; Uyar, T. Release and antibacterial activity of allyl isothiocyanate/ $\beta$-cyclodextrin complex encapsulated in electrospun nanofibers. Colloids Surf., B 2014, 120, $125-131$.

(20) Aytac, Z.; Kusku, S. I.; Durgun, E.; Uyar, T. Quercetin/ $\beta$ cyclodextrin inclusion complex embedded nanofibres: Slow release and high solubility. Food Chem. 2016, 197, 864-871.

(21) Aytac, Z.; Yildiz, Z. I.; Kayaci-Senirmak, F.; San Keskin, N. O.; Tekinay, T.; Uyar, T. Electrospinning of polymer-free cyclodextrin/ geraniol-inclusion complex nanofibers: enhanced shelf-life of geraniol with antibacterial and antioxidant properties. RSC Adv. 2016, 6, 46089-46099.

(22) Aytac, Z.; Yildiz, Z. I.; Kayaci-Senirmak, F.; San Keskin, N. O.; Kusku, S. I.; Durgun, E.; Tekinay, T.; Uyar, T. Fast-Dissolving, Prolonged Release, and Antibacterial Cyclodextrin/Limonene-Inclusion Complex Nanofibrous Webs via Polymer-Free Electrospinning. J. Agric. Food Chem. 2016, 64, 7325-7334.

(23) Aytac, Z.; Yildiz, Z. I.; Kayaci-Senirmak, F.; Tekinay, T.; Uyar, T. Electrospinning of cyclodextrin/linalool-inclusion complex nanofibers: Fast-dissolving nanofibrous web with prolonged release and antibacterial activity. Food Chem. 2017, 231, 192-201.

(24) Celebioglu, A.; Kayaci-Senirmak, F.; İpek, S.; Durgun, E.; Uyar, T. Polymer-free nanofibers from vanillin/cyclodextrin inclusion complexes: high thermal stability, enhanced solubility and antioxidant property. Food Funct. 2016, 7, 3141-3153.

(25) Higuchi, T. K.; Connors, A. Phase-solubility techniques. Adv. Analyt. Chem. Inst. 1965, 4, 117-212.

(26) Yalkowsky, S. H.; He, Y.; Jain, P. Handbook of aqueous solubility data; CRC press, 2016.

(27) Uyar, T.; Besenbacher, F. Electrospinning of uniform polystyrene fibers: The effect of solvent conductivity. Polymer 2008, $49,5336-5343$.

(28) Celebioglu, A.; Umu, O. C.; Tekinay, T.; Uyar, T. Antibacterial electrospun nanofibers from triclosan/cyclodextrin inclusion complexes. Colloids Surf., B 2014, 116, 612-619.

(29) Celebioglu, A.; Uyar, T. Cyclodextrin nanofibers by electrospinning. Chem. Commun. 2010, 46, 6903-6905.

(30) Celebioglu, A.; Uyar, T. Electrospinning of polymer-free nanofibers from cyclodextrin inclusion complexes. Langmuir 2011, 27, 6218-6226.

(31) Celebioglu, A.; Uyar, T. Electrospinning of nanofibers from non-polymeric systems: polymer-free nanofibers from cyclodextrin derivatives. Nanoscale 2012, 4, 621-631.

(32) Liu, L.; Zhu, S. Preparation and characterization of inclusion complexes of prazosin hydrochloride with $\beta$-cyclodextrin and hydroxypropyl- $\beta$-cyclodextrin. J. Pharm. Biomed. Anal. 2006, 40, $122-127$.
(33) Szente, L.; Szejtli, J. Highly soluble cyclodextrin derivatives: chemistry, properties, and trends in development. Adv. Drug Delivery Rev. 1999, 36, 17-28.

(34) Ciobanu, A.; Mallard, I.; Landy, D.; Brabie, G.; Nistor, D.; Fourmentin, S. Retention of aroma compounds from Mentha piperita essential oil by cyclodextrins and crosslinked cyclodextrin polymers. Food Chem. 2013, 138, 291-297.

(35) Antolovich, M.; Prenzler, P. D.; Patsalides, E.; McDonald, S.; Robards, K. Methods for testing antioxidant activity. Analyst 2002, 127, 183-198.

(36) Bortolomeazzi, R.; Sebastianutto, N.; Toniolo, R.; Pizzariello, A. Comparative evaluation of the antioxidant capacity of smoke flavouring phenols by crocin bleaching inhibition, DPPH radical scavenging and oxidation potential. Food Chem. 2007, 100, 14811489.

(37) Sharma, O. P.; Bhat, T. K. DPPH antioxidant assay revisited. Food Chem. 2009, 113, 1202-1205.

(38) Yanishlieva, N. V.; Marinova, E. M.; Gordon, M. H.; Raneva, V. G. Antioxidant activity and mechanism of action of thymol and carvacrol in two lipid systems. Food Chem. 1999, 64, 59-66.

(39) Nuchuchua, O.; Saesoo, S.; Sramala, I.; Puttipipatkhachorn, S.; Soottitantawat, A.; Ruktanonchai, U. Physicochemical investigation and molecular modeling of cyclodextrin complexation mechanism with eugenol. Food Res. Int. 2009, 42, 1178-1185. 